\title{
Throughput Measurement Method Using Command Packets for Mobile Robot Teleoperation Via a Wireless Sensor Network
}

\author{
Kei SAWAI \\ Department of Information and \\ Communication Engineering, Tokyo \\ Denki University \\ Tokyo, Japan
}

\author{
Ju Peng \\ Department of Information and \\ Communication Engineering, Tokyo \\ Denki University \\ Tokyo, Japan
}

\author{
Tsuyoshi Suzuki \\ Department of Information and \\ Communication Engineering, Tokyo \\ Denki University \\ Tokyo, Japan
}

\begin{abstract}
We are working to develop an information gathering system comprising a mobile robot and a wireless sensor network (WSN) for use in post-disaster underground environments. In the proposed system, a mobile robot carries wireless sensor nodes and deploys them to construct a WSN in the environment, thus providing a wireless communication infrastructure for mobile robot teleoperation. An operator then controls the mobile robot remotely while monitoring end-to-end communication quality with the mobile robot. Measurement of communication quality on wireless LANs has been widely studied. However, a throughput measurement method has not been developed for assessing the usability of wireless mobile robot teleoperation. In particular, a measurement method is needed that can handle mobile robots as they move around an unknown environment. Accordingly, in this paper, we propose a method for measuring throughput as a measure of communication quality in a WSN for wireless teleoperation of mobile robots. The feasibility of the proposed method was evaluated and verified in in practical field test where an operator remotely controlled mobile robots using a WSN.
\end{abstract}

Keywords-Wireless Sensor Networks; Rescue Robot Teleoperation; Communication Quality Measurement

\section{INTRODUCTION}

A wireless sensor network (WSN) would be useful for teleoperation of a mobile robot, but methods for measuring throughput between the operator and robot have not been argued enough. A common approach for throughput measurement is to calculate the maximum transfer amount per unit time, which provides the communication speed for delivery of payloads over the connection between sensor nodes. This method enables high-precision evaluation of throughput in networks where wireless communication quality is stable. However, throughput cannot be accurately measured in unstable networks where various types of noise occur. Furthermore, measurement by this method requires a few minutes, because many communication packets are sent. A rescue robot moves around and explores a disaster area, but such an environment contains debris and many obstacles leading to a risk of noise due to multipath fading of radio waves. Therefore, to operate the rescue robot promptly and smoothly, a rapid measurement method is necessary for evaluating the usability of rescue robot teleoperation.

Gathering information with a rescue robot in a disaster area is very important for assessing the situation, avoiding secondary disasters, and managing disaster mitigation [1]-[6]. In general, gathering information from a bird's eye view with an unmanned air vehicle is a useful method for ascertaining the situation in a disaster area. However, in an urban area with many underground spaces where information cannot be gathered from the air, the extent of the damage can be difficult to assess. Gathering information in underground spaces is important for avoiding secondary disasters [7]-[8] and planning rescue operations. When the communication infrastructure is damaged, cooperation among rescue workers is hindered by communication disconnection between aboveground and underground spaces, meaning that there is a high risk of rescue workers being involved in a secondary disaster, particularly in situations that are continuously or unexpectedly changing. Research on a disaster area information gathering system including WSN technology and rescue robots has recently been conducted considering past incidents in enclosed areas [7]-[8]. This system enables reduction of the risk of secondary disaster by employing a rescue robot rather than a human, and the WSN provides a wireless communication infrastructure for wireless rescue robot teleoperation. The WSN consists of many sensor nodes deployed by the robot and distributed spatially for cooperatively monitoring environmental conditions, such as temperature, sound, vibration, air pressure, and motion. The topology of the WSN changes automatically to optimize the communication path according to the communication quality between sensor nodes (Fig. 1). The rescue robot is defined as one of the sensor nodes constructing the WSN. Then, the WSN provides a wireless communication infrastructure where none existed before. Therefore, WSNs have been discussed as a method for gathering information and constructing communication infrastructure in disaster areas, and have been studied widely. Also, we have been investigating a robot wireless sensor network (RWSN) (Fig. 2) [9]-[13]. 


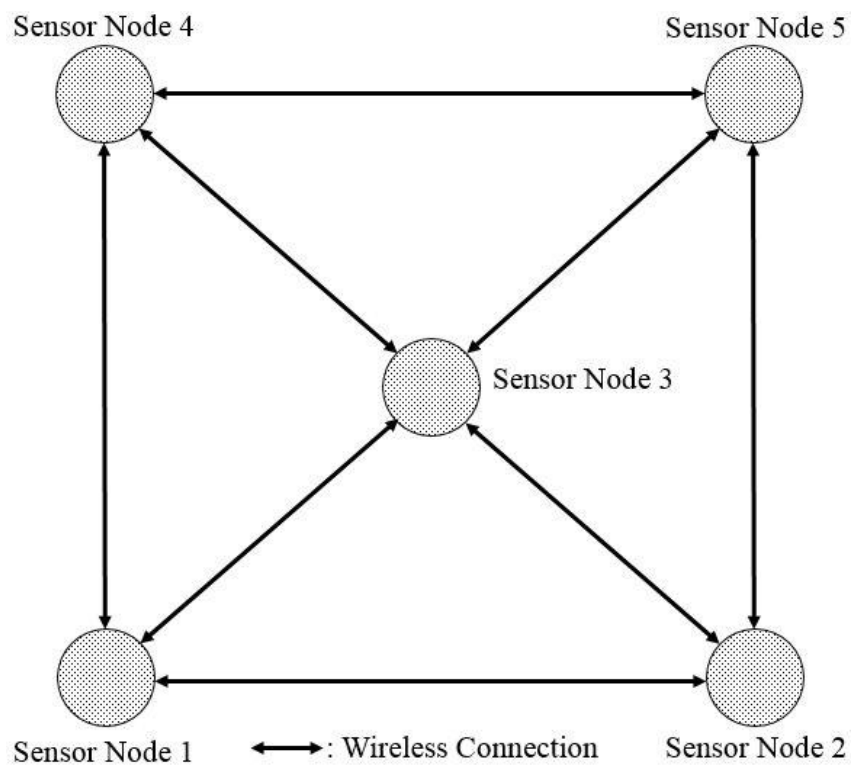

Fig. 1. Actively changed network topology of wireless sensor networks

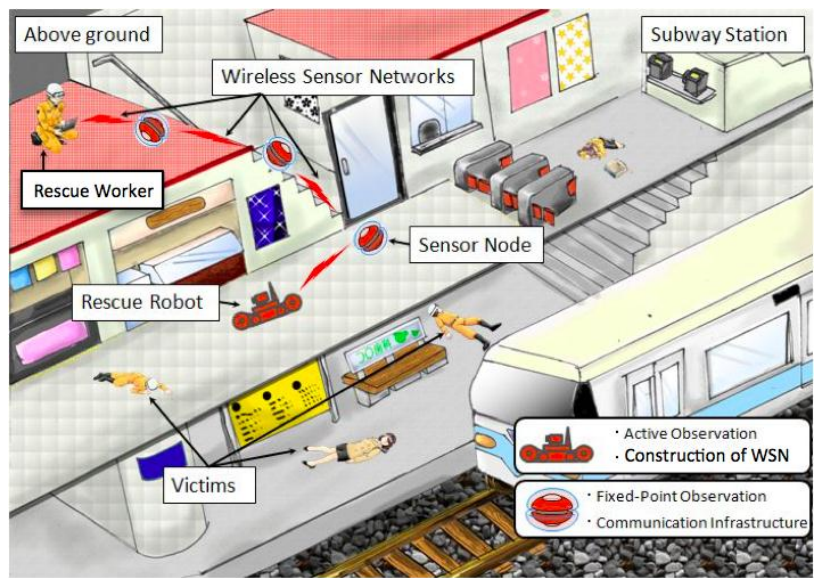

Fig. 2. Example deployment of a gathering disaster area information system using a WSN and rescue robot

However, in rescue robot teleoperation using a WSN, it is difficult to maintain communication connectivity between the operator and the rescue robot. Doing so requires measuring the throughput between the rescue robot and operator. Yet, the robot is a continually changing communication point because it moves around to gather information in the disaster area. The propagation of radio waves in a disaster area, such as an underground space, is difficult to estimate because of complicated building construction and damage caused by the disaster. Furthermore, the communication distance between the rescue robot and the adjacent sensor node in an ad hoc network changes as the robot moves around, and so the electric field signal strength between the rescue robot and adjacent sensor node also fluctuates. For these reasons, existing methods cannot perform accurate measurement of throughput between the rescue robot and operator in an unstable communication environment such as a post-disaster underground space.

Most existing methods for measuring throughput assume a normal living space as the environment for the WSN, but this assumption does not hold for a post-disaster underground space. Furthermore, the existing methods are not intended for networks with a continually moving sensor node such as a rescue robot. In an RWSN, accurate measurement of throughput cannot be performed because the moving sensor node continually changes the communication point. For existing method to measure throughput accurately, many packets must be sent from the measurer to the measurement object, and then the throughput is calculated by using the size and number of packets received by the measurement object. Sending and receiving packets requires a few minutes, which reduces the usability of rescue robot teleoperation. When such a method is used, the operator cannot control the rescue robot during throughput measurement because many communication packets are sent instead of control packets. If the robot cannot communicate over the network, it could become uncontrollable and cause a secondary disaster. To prevent network disconnection while deploying sensor nodes, during each interval the operator must stop the rescue robot by utilizing command packets and then measure throughput as indicator of the strength of wireless communication connectivity between the rescue robot and operator. Thus, existing methods have the problem of reducing the practical utility of the disaster area information gathering system. Furthermore, in research on mobile robots and WSN systems, the communication connectivity of the system has not been defined clearly [14][17]. Most studies adopt received signal strength indicator (RSSI) as a measure of wireless communication connectivity to maintain the network configuration of the system, and RSSI is measured by an RF module over a very short time [18]-[26]. However, RSSI indicates communication quality in the physical layer and cannot evaluate communication quality in the transport layer for sending and receiving communication packets. For example, even if the RSSI level is good, a communication environment with strong radio wave interference or absorption will have reduced throughput. Thus, RSSI cannot correctly indicate the strength of communication connectivity. Quick evaluation of communication connectivity with high precision is necessary for monitoring packet traffic in the transport layer.

Accordingly, here we considered an approach to reduce the time required for measuring throughput between the rescue robot and the operator by using the command packets for robot control, rather than communication packets. The proposed system uses a TCP/IP-compliant communications protocol and an IEEE 802.11 wireless LAN protocol that are compatible with teleoperation of a rescue robot. The proposed method was actually implemented using a WSN and rescue robot in a field test to examine the feasibility of the measurement time reduction. The next section describes the RWSN of our proposed disaster area information gathering system comprising a rescue robot and WSN. Section 3 presents details of the proposed throughput measurement method, and then field test results for performance evaluation are presented in Sections 4 and 5. Conclusions are given in Section 6.

\section{RoBot WIRELESS SENSOR NETWORKS}

An RWSN is constructed from a WSN by using a rescue robot that deploys sensor nodes along its movement path. In our sensor node deployment method for constructing the WSN, the rescue robot delivers sensor nodes that were connected in 
advance and configured with a defined routing path. Thus, the rescue robot delivers all the sensor nodes with a defined routing path into an underground space along the robot's movement path. Deployed sensor nodes communicate with adjacent sensor nodes wirelessly, and as sensor nodes are deployed, the wireless communication distance between the operator and the rescue robot is extended via the WSN in the underground space. The operator controls the rescue robot through the communication infrastructure provided by the constructed WSN. In the network topology of the RWSN, sensor nodes are linearly connected to prevent communication errors due to auto-routing control (Fig. 3, Fig. 4).

Generally, the WSN can automatically decide the routing path of data transfer by various means, including the RSSI between adjacent sensor nodes, the time difference of arrival of communication packets, the end-to-end throughput, or the packet loss rate. The routing paths in the WSN are reconstructed according to changes in communication quality. However, repeated reconstruction of the routing path causes communication disconnection and reconnection between sensor nodes, a situation that causes serious problems in the teleoperation of mobile robots. The rescue robot cannot establish a wireless communication infrastructure by an existing method because the risk of disconnection between rescue robot and adjacent sensor node is high. Teleoperation with irregular interruptions of wireless communication degrades the usability of the rescue robot, and so information gathering performance degrades as well. Such a situation must be avoided to prevent the robot from becoming uncontrollable and creating a risk of secondary disaster. Furthermore, communication quality strongly varies according to secondary disaster damages in the disaster area. Therefore, the routing path is not repeatedly reconstructed in the WSN.

In the proposed RWSN system, it is necessary to maintain communication connectivity in order to predict disconnection between a sensor node to be deployed and the adjacent sensor node. To predict disconnection when extending the WSN, the change in throughput should be monitored between the sensor node to be deployed and the adjacent sensor node. End-to-end throughput between the operator and the rescue robot is known by connecting all sensor nodes in advance. Maintaining this throughput stabilizes end-to-end communication connectivity, and then it is necessary to maintain the throughput value between adjacent sensor nodes. An IEEE 802.11 wireless LAN module can control throughput speed automatically by monitoring RSSI, allowing the throughput to be decreased when RSSI drops below a defined threshold. Thus, in order to maintain throughput between a sensor node to be deployed and the adjacent sensor node, it is necessary to monitor the RSSI value at the same time as throughput measurement. Maintaining throughput with RSSI monitoring stabilizes endto-end communication connectivity in the RWSN system. Regarding elemental technologies for an RWSN information gathering system in post-disaster underground spaces, we have previously reported details of the RWSN, development of impact-resistant sensor node, a rescue robot with a sensor node deployment mechanism, and an RWSN construction method that employs a rescue robot with WSN technology. In particular, we have demonstrated the importance of communication connectivity between deployed sensor node and adjacent sensor nodes.

In this paper, the proposed method to reduce throughput measurement time is implemented and evaluated on the assumption that the RWSN is already constructed in the target environment. Details of this method are described in the next section.

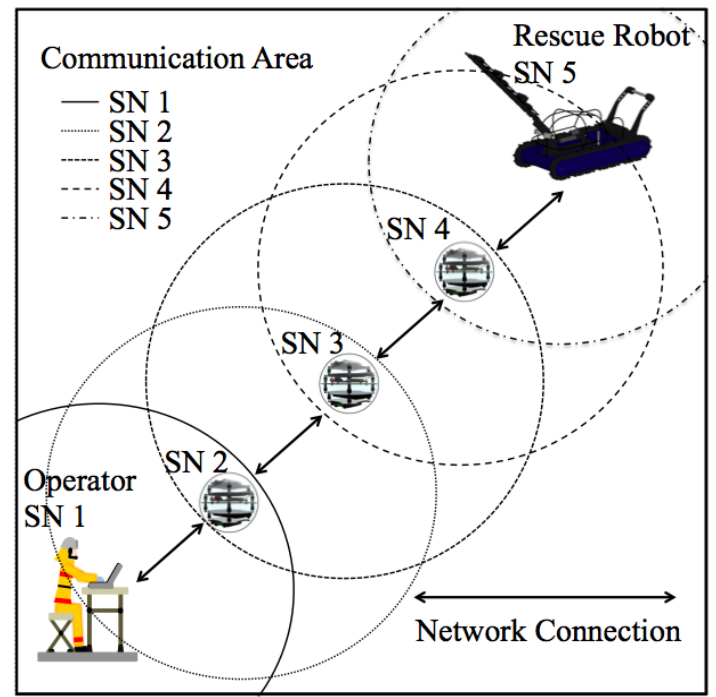

Fig. 3. Teleoperation of a rescue robot in an RWSN (SN: sensor node)

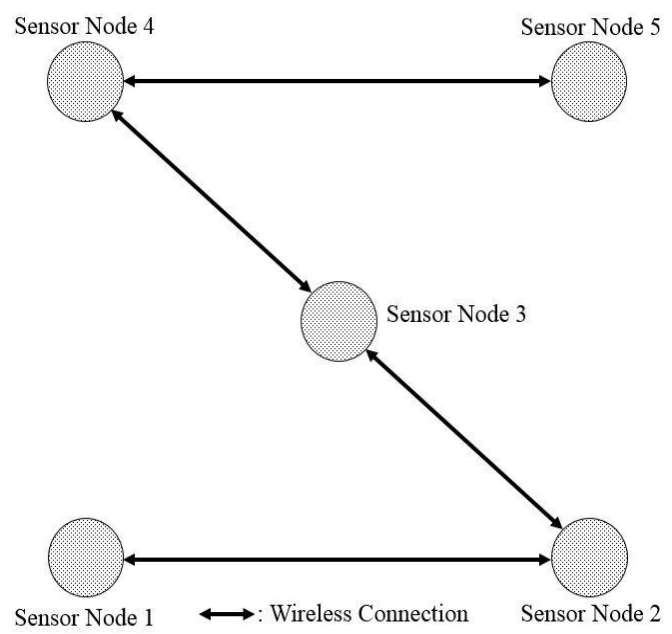

Fig. 4. Network topology of the RWSN

\section{ThroughPUt MEASUREMENT METHOD USING COMMAND PACKET FOR TELEOPERATION OF RESCUE ROBOT}

\section{A. Assumed Environment for Constructing the WSN}

We assume that in a post-disaster underground space, it is necessary to construct the disaster area information gathering system on the first basement floor between the exit and entrance. In particular, this environment is configured by two EXIT stairs, and $30 \mathrm{~m}$ path between them. In the Japanese Building Standards Law, $30 \mathrm{~m}$ is specified as the maximum permissible distance between EXIT stairs for escaping to the ground from underground structures. Moreover, most paths in underground spaces are designed to be straight in consideration 
of emergency evacuation. Therefore, we assume that RWSN is constructed in this environment, and the design of the proposed method is based on these environmental conditions (Fig. 5).

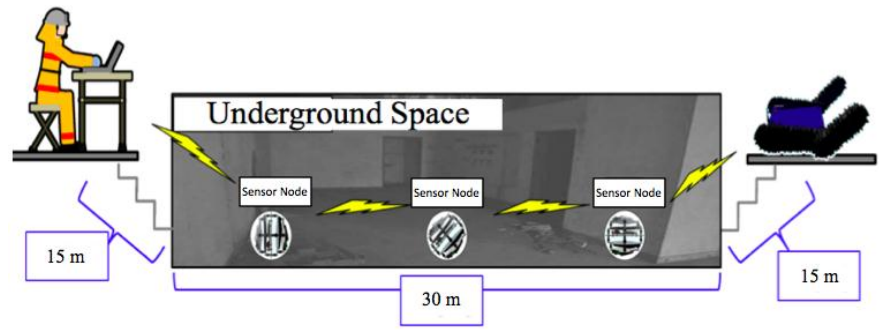

Fig. 5. Assumed environment for constructing the RWSN

\section{B. Throughput Measurement Method without Interruption of Communication for Rescue Robot Teleoperation}

We propose a throughput measurement method to reduce measurement time by measuring small command packets that are sent and received for mobile robot teleoperation (Fig. 6). The operator sends command packets configured from action parameters for moving and sensing, and then the mobile robot sends back status information based on captured image data, temperature, movement speed, acceleration, and so forth. These communications between the operator and the rescue robot should be continued without interruption to maintain the operability of teleoperation. Even when the operator does issue a command to the rescue robot, packets are continuously sent and received by the operator and the rescue robot in the background to predict communication disconnection and an uncontrollable status of the rescue robot. Generally, teleoperation systems use small packets for stable communication quality over a network.

Therefore, we propose a throughput measurement method that uses these command packets. Throughput is the amount of transferred data per unit time, and is a measure of communication speed. Throughput $\mathrm{T}$ [bps] is calculated by using equation (1).

$$
T=\frac{8 B}{t}
$$
time.

Here, B [byte] is the packet size and $t$ [s] is the transfer

The size of command packets for teleoperation of the rescue robot is defined as described above. Then, ID numbers are consecutively assigned to the packets as they are sent to the operator or the rescue robot. Furthermore, the transmission interval between packets is constant. Thus, in the proposed measurement method, throughput is calculated by equation (1). By using command packets in this way, it is not necessary to interrupt teleoperation of the rescue robot teleoperation or to send many communication packets for throughput measurement as in existing method. Moreover, the operator can obtain the throughput within a few seconds by the proposed method, whereas the existing method takes a few minutes for throughput measurement. In a network system implementing various network applications, existing methods measure the maximum value of throughput between terminals by sending many communication packets, which preload communication band to the utmost remits in the network.
However, a network system that uses only a defined network application such as disaster area information gathering system does not have to maximize throughput. To maintain communication connectivity, it is important to monitor the change in throughput between terminals. Although the maximum throughput value cannot be measured, the proposed method can evaluate the throughput stability more quickly than the existing method. Therefore, we consider our approach more suitable than existing methods for systems such as the disaster area information gathering system in post-disaster underground space.

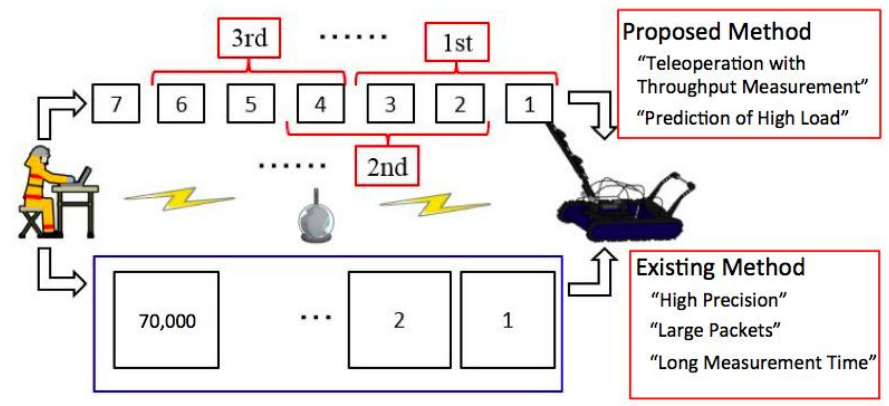

Fig. 6. Comparison of existing and proposed throughput measurement methods

\section{Definition of Packet Size}

To measure an aspect of communication quality such as throughput, it is important to define packet size. If the packet size is too small, the packet transfer time is adversely affected from noise due to network traffic. If the packet size is too big, a transferred packet is automatically divided into multiple packets by TCP/IP and UDP. This mechanism is defined as IP fragmentation, in which transferred packets are divided in order to pass a link where the maximum transmission unit is smaller than the original datagram size. However, this mechanism changes the transmission time of packets between terminals. Therefore, in our proposed method, we adopt a packet size of 1,400 bytes to prevent IP fragmentation.

\section{Packet Transfer Method in the Proposed Throughput Measurement}

The proposed packet transfer method transfers multiple packets to an extent that does not adversely affect the communication path. Throughput measurement by command packet transfer can be affected by various types of noise along the network path, and so the throughput is inaccurate due to transfer of packets, changes in the surrounding environment, and electrical noise. In particular, the building structures in a post-disaster environment continually change as a result of secondary disaster damage, and the features of the radio wave propagation environment also continuously changes. Thus, in the throughput measurement method, multiple packets are transferred to predict the influence of noise along the communication path, and the overall average of all received packets at a determined time is taken as the measurement result. Throughput measured in this way takes into consideration environmental noise, and can be used for continuous monitoring of communication connectivity between terminals. Generally, a teleoperation system of a rescue robot continuously communicates in order to improve the usability of 
teleoperation between the operator and rescue robot by sending and receiving command packet. Therefore, the throughput measurement method using continuously transfer of command packets on the communication path can be widely implemented in teleoperation systems of rescue robots. As described in the next section, we conducted a field test to evaluate the proposed throughput measurement method using an actual rescue robot and WSN.

\section{PERFORMANCE EVAlUation OF THROUGHPUT MEASUREMENT METHOD USING COMMAND PACKETS CONTROLLING RESCUE RoBOT}

\section{A. Performance Evaluation}

This section presents a performance evaluation of our proposed throughput measurement method. We measured throughput by using an existing method and the proposed method, and then experimental results are verified in a comparative evaluation. In the experiment, an RWSN is adopted as a disaster area information gathering system in a post-disaster underground space.

\section{B. Experimental Condition}

The RWSN used in this experiment was constructed by a rescue robot deploying sensor nodes. The rescue robot was equipped with three sensor nodes and deployed them at $15 \mathrm{~m}$ intervals. Thus, the RWSN was intended to span $60 \mathrm{~m}$, and the WSN provided a wireless communication infrastructure with a communication path of this length. The operator used the RWSN to control the rescue robot, and the RWSN construction was based on a sensor node deployment method that we proposed in the past. The sensor node deployment method was provided to predict network disconnection when constructing the RWSN. In the experiment, the proposed method defined the number of transferred packets to be used for a single measurement as 40 packets in consideration of packet traffic noise. Among existing methods comparable to the proposed method, "utest" (NTTPC Communications Ltd.) was adopted, which measures an accurate value of throughput by sending $1,000,000$ packets each of 1500 bytes for a single measurement. In each of the existing method and the proposed method, measurements were performed 10 times, and then the overall mean was calculated.

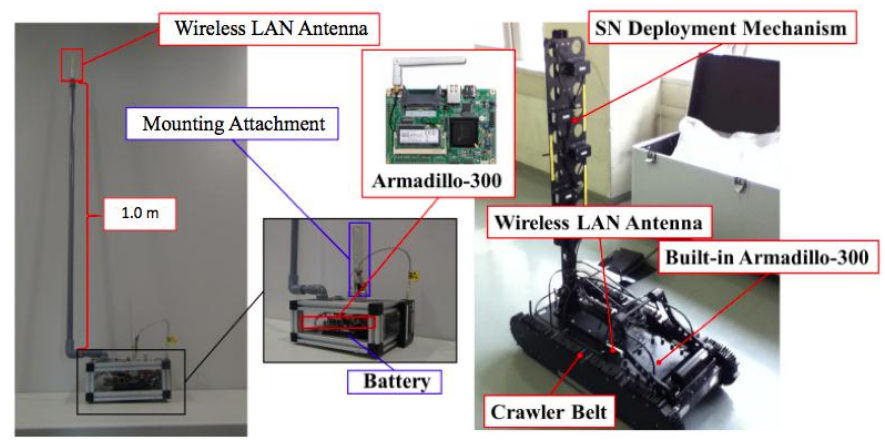

(a) Developed sensor node (b) Rescue robot with sensor node deploying mechanism

Fig. 7. Sensor node and rescue robot using in constructing RWSN

To construct the WSN, we adopted the sensor node device developedin our previous studies (Fig. 7 (a)). The sensor node is equipped with a CPU board, memory device, CompactFlash disc drive, IEEE $802.11 \mathrm{~b} / \mathrm{g}$ wireless LAN module, a digital camera, an A/D converter, and a battery. The sensor nodes are controlled by software implemented in Linux (Debian). In this way, a WSN was constructed by utilizing the AODV-uu protocol for ad hoc networks. Table 1 shows the specification of our developed sensor node. As shown in Fig. 7 (b), a crawler-type mobile robot (S-90LWX, Topy Industries, Ltd. was used as the rescue robot in this experiment. A sensor node deployment mechanism was developed for WSN construction and installed on the rescue robot; this mechanism can hold five sensor nodes using five solenoid-operated locks. Figure 8 shows the framework of this mobile robot with the sensor node deployment mechanism. Then, the operator can control the crawler robot and the deployment mechanism remotely by utilizing TCP/IP and UDP. The experiment is performed in the passageway with a length of 300 [m] or more in Tokyo Denki University. Figure 9 (a), (b) shows experimental overview in this performance evaluation.

TABLE I. SPECIFICATIONS OF SENSOR NODES FOR CONSTRUCTING THE RWSN

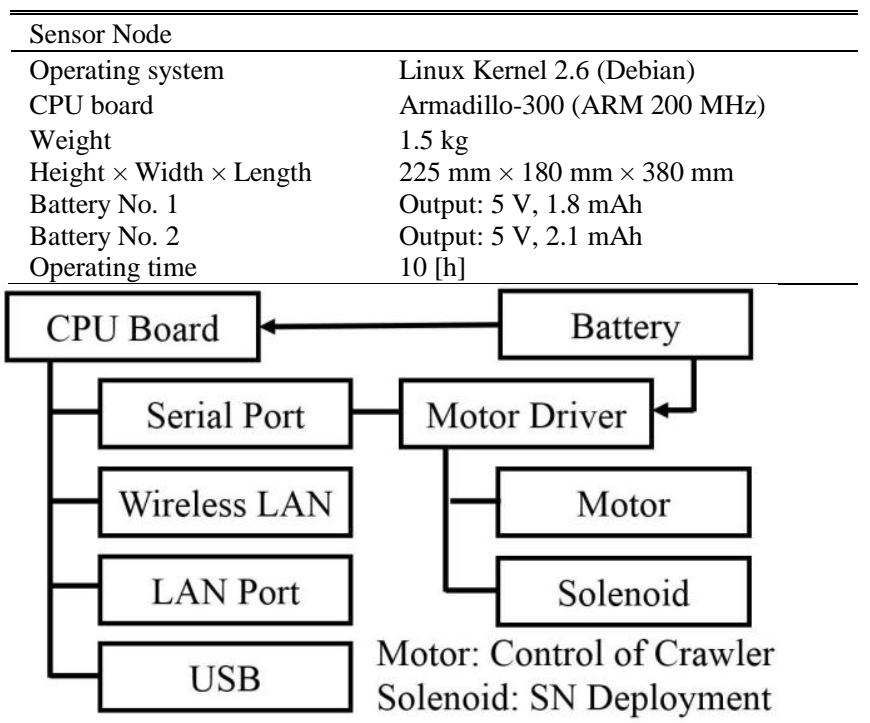

Fig. 8. Configuration of the rescue robot with sensor node deployment mechanism
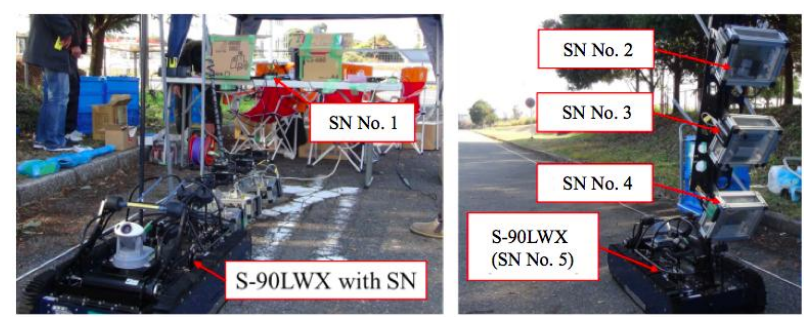

(a) Experimental Condition

(b) Rescue Robot mounting SNs

Fig. 9. Scenes from performance evaluation field test

\section{Experimental Result}

Figure 10 shows the experimental results. Figure 11 shows a comparative evaluation of error range. Throughput values in both the existing method (utest) and the proposed method showed similar trends in the performance evaluation test. The 
proposed method gave slightly higher throughput values than utest, showing that the proposed method did not slow down packet traffic.

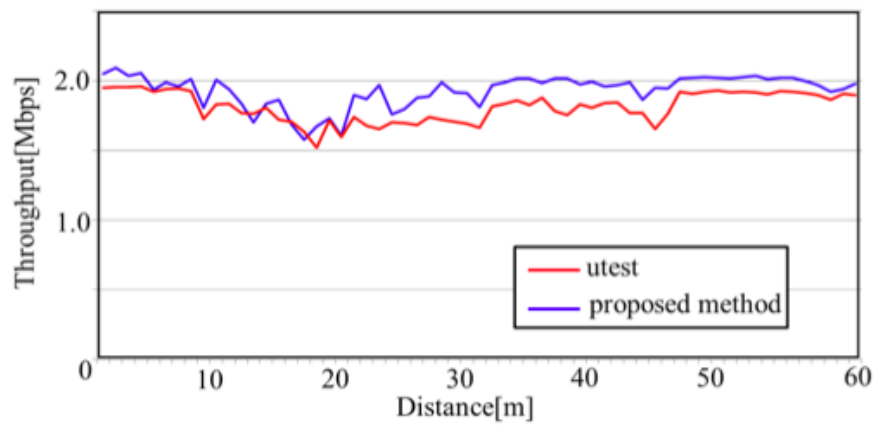

Fig. 10. Experimental Results Using "utest" and Proposed Method

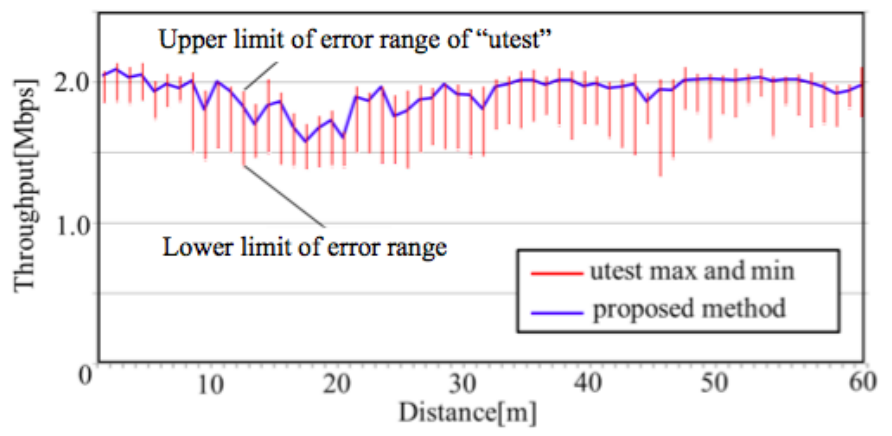

Fig. 11. Comparative Evaluation of Error Range

\section{DISCUSSION}

The correlation coefficient between the throughputs measured by the utest and the proposed method was 0.8655 , showing that there was a high correlation in this performance evaluation. Overall mean values of throughput measured by the proposed method were slightly higher than the mean values measured by utest, because the proposed method did not produce excessive packet traffic on the communication path. However, all throughputs measured by the proposed method were within the upper limit of the error range of measurements by utest. Thus, we confirmed that the throughput values obtained by the proposed method were correct. Furthermore, this proposed method enabled to control of the rescue robot by a remote operator. These results show the applicability of the proposed method to RWSN.

\section{CONCLUSION}

In this paper, we proposed a method for reducing the time required for measuring the throughput between a rescue robot and an operator in a constructed WSN with the aim of improving the usability of a disaster area information gathering system in post-disaster underground spaces. This method was designed to avoid excessive sending of communication packets as in existing methods and to suit an assumed underground disaster environment. The proposed method was designed to utilize the command packets for controlling the rescue robot and used a wireless LAN IEEE 802.11 protocol compatible with wireless teleoperation of a rescue robot. The developed method was implemented in a rescue robot and WSN, and then feasibility of the proposed method was confirmed on the basis of measurement error and measurement time from the results of a performance evaluation field test for comparison with utest. Furthermore, throughput measurement using the proposed method allowed the throughput to be measured without stopping the robot.

In future work, we plan to improve the graphical user interface to display throughput to the operator while controlling the rescue robot. Also, we will consider a method for measuring packet jitter between the rescue robot and operator for implementing a video streaming function that uses the rescue robot's camera to gather disaster area information in a post-disaster underground space.

\section{ACKNOWLEDGMENT}

This work was partially supported by MEXT KAKENHI Grant Number 15631330.

We are grateful to Mr. Shigeaki Tanabe and Mr. Ryuta Kunimoto who were graduate students in Tokyo Denki University in 2011 and 2012 respectively for their support in the RWSN system development.

\section{REFERENCES}

[1] CHI Hao-yuan, LIU Xu, XU Xiao-dong, "A Framework for Earthquake Disaster Mitigation System," Proceedings of 2011 China located International Conference on Information Systems for Crisis Response and Management (ISCRAM), pp.490-495, 2011.

[2] Huang AN, "China's Emergency Management Mechanisms for Disaster Prevention and Mitigation," Proceedings of International Conference on E-Business and E-Government (ICEBEG), pp.2403-2407, 2010.

[3] Yoshiaki KANAEDA, Kazushige MAGATANI, "Development of the device to detect SPO2 in the Field,"31st Annual International Conference of the IEEE EMBS, pp.412-415, September 2009.

[4] Y. Kawata, "Disaster Mitigation due to next Nankai earthquake tsunami occurring in around 2035," Proceedings of International Tsunami Symposium 2001, session 1, pp. 315-329, 2001.

[5] Y. Kawata, "The great Hanshin-Awaji earthquake disaster: damage, social response, and recovery," Journal of Natural Disaster Science, Vol. 17, No. 2, pp.1-12, 1995.

[6] H. Kawakata, Y. Kawata, H. Hayashi, T. Tanaka, K. C. Topping, K. Yamori, P. Yoshitomi, G. Urakawa and T. Kugai, "Building an integrated database management system of information on disaster hazard, risk, and recovery process," Annuals of Disas. Prev. Res. Inst., Kyoto Univ., No.47 C, 2004.

[7] Abishek T K, Chithra K R and Maneesha V. Ramesh, "ADEN:Adaptive Energy Efficient Network of Flying Robots Monitoring over Disaster Hit Area," Proceedings of 8th IEEE International Conference on Distributed Computing in Sensor Systems (IEEE DCOSS), pp.306-310, 2012.

[8] Abishek T K, Chithra K R, Maneesha V Ramesh, "AER: Adaptive Energy Efficient Routing Protocol For Network of Flying Robots Monitoring over Disaster Hit Area," Proceedings of 21st Annual Wireless and Optical Communi-cations Conference (WOCC), pp.166169, 2012.

[9] K. Sawai, T. Suzuki, H. Kono, Y. Hada and K. Kawabata, "Development of a SN with impact-resistance capability for gathering disaster area information," 2008 Internatio-nal Symposium on Nonlinear Theory and its Applications (NOLTA2008), pp.17-20, 2008.

[10] Tsuyoshi Suzuki, Kei Sawai, Hitoshi Kono and Shigeaki Tanabe, "Sensor Network Deployment by Dropping and Throwing Sensor Node to Gather Information Underground Spaces in a Post-Disaster Environment," Descrete Event Robot, iConcept PRESS, in Press. 2012.

[11] K. Sawai, H. Kono, S. Tanabe, K. Kawabata, T. Suzuki, "Design and Development of Impact Resistance Sensor Node for Launch Deployment into Closed Area," In international journal of sensing for 
industry(Sensor Review), Emerald Group Publishing Ltd., Vol. 32, pp.318-326, 2012.

[12] S. Tanabe, K. Sawai and T. Suzuki, "Sensor Node Deployment Strategy for Maintaining Wireless Sensor Network Communication Connectivity," International Journal of Advanced Computer Science and Applications ( IJACSA ), The Science and Information organization, Vol.2, No. 12, pp.140 - 146, 2011.

[13] H. Sato, K. Kawabata and T. Suzuki, "Information Gathering by wireless camera node with Passive Pendulum Mechanism," International Conference on Control, Automation and Systems 2008 (ICCAS2008), pp.137-140, 2008.

[14] T. Yoshida, K. Nagatani, E. Koyanagi, Y. Hada, K. Ohno, S. Maeyama, H. Akiyama, K. Yoshida and Satoshi Tadokoro, "Field Experiment on Multiple Mobile Robots Conducted in an Underground Mall," Field and Service Robotics Springer Tracts in Advanced Robotics, vol. 62, pp365$375,2010$.

[15] H. Jiang, J. Qian, and W. Peng, "Energy Efficient Sensor Placement for Tunnel Wireless Sensor Network in Underground Mine," Proceedings of 2nd International Conference on Power Electronics and Intelligent Transportation System(PEITS 2009), pp. 219-222, 2009.

[16] J. Xu, S. Duan and M. Li, "The Research of New Type Emergency Rescue Communication System in Mine Based on Wi-Fi Technology," Proceedings of IEEE 3rd International Conference on Communication Software and Networks (ICCSN), pp. 8-11, 2011.

[17] K. Nagatani, S. Kiribayashi, Y. Okada, K. Otake, K. Yoshida, S. Tadokoro, T. Nishimura, T. Yoshida, E. Koyanagi, M. Fukushima and S. Kawatsuma, "Emergency Response to the Nuclear Accident at the Fukushima Daiichi Nuclear Power Plants using Mobile Rescue Robots," Journal of Field Robotics, vol. 30, no. 1, pp. 44-63, 2013.

[18] Parker, E., L., Kannan, B., Xiaoquan, F., Yifan, T. (2003). Heterogeneous Mobile Sensor Net Deployment Using Robot Herding and Line of Sight Fromations, Proceedings of 2003 IEEE/RSJ International Conference on Intelligent Robots and Systems (IROS2003), Volume 3. pp.2488-2493, 2003.
[19] T. Umeki, H. Okada, K. Mase, "Evaluation of Wireless Channel Quality for an Ad Hoc Network in the Sky SKYMESH," Proceedings of Sixth International Symposium on Wireless Communication Systems 2009 (ISWCS'09). pp.585-589, 2009.

[20] Helge-Bjorn Kuntze, Christian W. Frey, Igor Tchouchenkov, Barbara Staehle, Erich Rome, Kai Pfeiffer, Andreas Wenzel and Jurgen Wollenstein, "SENEKA - Sensor Network with Mobile Robots for Disaster Management," Homeland Security (HST), pp.406-410, 2012.

[21] E. Budianto, M.S. Alvissalim, A. Hafidh, A. Wibowo, W. Jatmiko, B. Hardian, P. Mursanto and A. Muis, "Telecommunication Networks Coverage Area Expansion in Disaster Area using Autonomous Mobile Robots : Hardware and Software Implementation," Proceedings of International Conference on Advanced Computer Science and Information Systems (ICACSIS), pp.113-118, 2011.

[22] Andrew Chiou, and Carol Wynn, "Urban Search and Rescue Robots in Test Arenas: Scaled Modeling of Disasters to Test Intelligent Robot Prototyping," Proceedings of International Conference on Autonomic and Trusted Computing (ATC), pp.200-205, 2009.

[23] A. Howard, J. Matric, and J. Sukhatme, "Mobile Sensor Network Deployment using Potential Fields," A Distributed Scalable Solution to the Area Coverage Problem, Distributed Autonomous Robotics Systems 5, Springer-Verlag. pp.299-308, 2002.

[24] Wing-Yue Geoffrey Louie, and Goldie Nejat, "A victim identification methodology for rescue robots operating in cluttered USAR environments," Advanced Robotics, vol. 27, issue. 5, pp. 373-384, 2013.

[25] Andrew Markham and Niki Trigoni, "Magneto-Inductive NEtworked Rescue System (MINERS):Taking Sensor Networks Underground," Proceedings of the 11th international conference on Information Processing in Sensor Networks (IPSN '12), pp. 317-328, 2012.

[26] Josh D. Freeman, Vinu Omanan, and Maneesha V. Ramesh, "Wireless Integrated Robots for Effective Search and Guidance of Rescue Teams," Proceedings of 8th International Conference on Wireless and Optical Communications Networks (WOCN 2011), pp. 1-5, 2011. 